\title{
RESEARCH
}

Open Access

\section{Investigation of reactive astrogliosis effect on post-stroke cognitive impairment}

Kuo-Lun Huang ${ }^{1 \dagger}$ (D), Ing-Tsung Hsiao ${ }^{2,3 \dagger}$, Meng-Yang Ho ${ }^{1,4}$, Jung-Lung Hsu ${ }^{5,6}$, Yeu-Jhy Chang ${ }^{1}$, Ting-Yu Chang ${ }^{1}$, Chi-Hung Liu', Chien-Hung Chang ${ }^{1}$, Yi-Ming Wu', Kuan-Yi Wu', Shiaw-Pyng Wey ${ }^{2,3}$, Tzu-Chen Yen ${ }^{2,3}$, Nobuyuki Okamura ${ }^{9,10}$, Tsong-Hai Lee ${ }^{1 *}$ and Kun-Ju Lin ${ }^{2,3^{*}}$

\begin{abstract}
Background: The aim of this study is to investigate the associations between post-stroke cognitive impairment (PSCI) severity and reactive astrogliosis (RA) extent on normalized ${ }^{18} \mathrm{~F}$-THK-5351 positron-emission tomography (PET) imaging in amyloid-negative patients with first-ever stroke.

Methods: We prospectively enrolled 63 amyloid-negative patients with first-ever stroke. Neurocognitive evaluation, MRI, ${ }^{18} \mathrm{~F}$-THK-5351, and ${ }^{18} \mathrm{~F}$-florbetapir PET were performed around 3 months after stroke. The ${ }^{18} \mathrm{~F}$-THK-5351 uptake intensity was normalized using a signal distribution template to obtain the Z-SUM scores as the RA extent in the whole brain and cerebral hemisphere ipsilateral to stroke lesion. We evaluated stroke volume, leukoaraiosis, and brain atrophy on MRI. We used a comprehensive neurocognitive battery to obtain composite cognitive scores, and defined PSCl as a general cognitive function score $<-1$. We analyzed the influence of Z-SUM scores on PSCl severity after adjusting for demographic, vascular, and neurodegenerative variables.

Results: Twenty-five of 63 stroke patients had PSCI. Patients with PSCI had older age, lower education, and more severe cortical atrophy and total Z-SUM scores. Total Z-SUM scores were significantly associated with general cognitive and executive functions at multiple regression models. Path analyses showed that stroke can exert cognitive influence directly by stroke itself as well as indirectly through RA, including total and ipsilateral Z-SUM scores, in patients with either right or left hemisphere stroke.

Conclusion: The patterns and intensity of ${ }^{18} \mathrm{~F}$-THK-5351 uptake in amyloid-negative patients with first-ever stroke were associated with PSCI manifestations, which suggests that RA presents a modulating effect in PSCl development.
\end{abstract}

Keywords: Post-stroke cognitive impairment, Reactive astrogliosis, PET, Stroke

\footnotetext{
* Correspondence: thlee@adm.cgmh.org.tw; kunjulin@gmail.com

${ }^{\dagger}$ Kuo-Lun Huang and Ing-Tsung Hsiao contributed equally to this work. 'Department of Neurology, Linkou Chang Gung Memorial Hospital, and College of Medicine, Chang Gung University, No. 5, Fuxing St., Guishan, Taoyuan, Taiwan

${ }^{2}$ Department of Nuclear Medicine and Molecular Imaging Center, Linkou Chang Gung Memorial Hospital, No. 5, Fuxing St., Guishan, Taoyuan, Taiwan Full list of author information is available at the end of the article
}

(c) The Author(s). 2020 Open Access This article is licensed under a Creative Commons Attribution 4.0 International License, which permits use, sharing, adaptation, distribution and reproduction in any medium or format, as long as you give appropriate credit to the original author(s) and the source, provide a link to the Creative Commons licence, and indicate if changes were made. The images or other third party material in this article are included in the article's Creative Commons licence, unless indicated otherwise in a credit line to the material. If material is not included in the article's Creative Commons licence and your intended use is not permitted by statutory regulation or exceeds the permitted use, you will need to obtain permission directly from the copyright holder. To view a copy of this licence, visit http://creativecommons.org/licenses/by/4.0/ The Creative Commons Public Domain Dedication waiver (http://creativecommons.org/publicdomain/zero/1.0/) applies to the data made available in this article, unless otherwise stated in a credit line to the data. 


\section{Introduction}

Post-stroke dementia (PSD) and post-stroke cognitive impairment (PSCI) affect up to one-third of stroke survivors and mostly occur within the first 6 months $[1,2]$. PSD is not a single disease entity; rather, it describes an unspecified dementia syndrome that occurs after stroke [3]. Epidemiological studies have reported PSD etiologies to be attributed to Alzheimer's disease (AD) or co-occurring $\mathrm{AD}$ and vascular dementia in $29 \%$ to $61 \%$ of patients with PSD [4]. According to the double-hit theory on PSCI development, patients with stroke have different post-stroke cognitive trajectories depending on their amyloid plaque burden and neuroinflammation severity [5]. Functional and molecular imaging has facilitated the understanding of the complex interactions between neurodegeneration and vascular injury in post-stroke cognitive presentations [6]. On the other hand, stroke, like other CNS injury, will induce a cascade of neuroinflammatory responses, and neuroinflammatory fluid biomarkers, such as C-reactive protein, IL-8 and IL-12, have been identified to be associated with PSCI occurrence $[7,8]$. However, there is limited human neuroimaging evidence on the relationships among stroke lesions, neuroinflammatory severity, and PSCI [5, 9].

${ }^{18} \mathrm{~F}-\mathrm{THK}-5351$ is a radiotracer designed for in vivo tau protein detection in patients with $\mathrm{AD}$ [10]. In addition to tau protein binding, ${ }^{18} \mathrm{~F}-\mathrm{THK}-5351$ has been reported to have an affinity for monoamine oxidase-B (MAO-B), which might explain the off-target ${ }^{18} \mathrm{~F}$-THK-5351 binding in the striatum, thalamus, and brainstem [11, 12]. Furthermore, MAO-B is largely expressed in astrocytes during the neuroinflammatory phenomenon of reactive astrogliosis (RA) [13]. Previous studies have reported increased ${ }^{18} \mathrm{~F}-\mathrm{THK}-5351$ uptake around stroke lesions, which might provide information regarding astrocyte-related neuroinflammatory changes $[14,15]$. However, stroke-induced ${ }^{18}$ F-THK-5351 uptake signals might overlap with background signals, mainly in striatum and thalamus, thereby hindering the quantification of stroke-induced neuroinflammation on ${ }^{18} \mathrm{~F}-\mathrm{THK}-5351$ positron-emission tomography (PET) imaging.

Firstly, we aimed to establish a signal distribution template of ${ }^{18} \mathrm{~F}$-THK-5351 PET imaging using healthy subjects, and subsequently apply the template in patients with stroke to diminish subcortical background signals through statistical transformation. Thereby, strokeinduced ${ }^{18} \mathrm{~F}$-THK-5351 uptake could be quantified as a neuroinflammatory imaging biomarker of RA. Secondly, we aimed to explore the correlations between PSCI and neuroinflammation severity on ${ }^{18} \mathrm{~F}-\mathrm{THK}-5351$ PET imaging in amyloid-negative patients with first-ever stroke.

\section{Materials and methods}

\section{Participants}

We conducted a prospective, cross-sectional study to screen 72 patients with recent first-ever stroke (around 3 months after onset, median 87 days with interquartile range from 75 to 100 days) from the Department of Neurology and Stroke Center at Linkou Chang Gung Memorial Hospital, Taiwan, as previously described [15]. These stroke patients fulfilled the inclusion criteria: (1) a diagnosis of ischemic or hemorrhagic stroke confirmed on brain computed tomography (CT) or magnetic resonance imaging (MRI) at stroke onset; (2) no history of old stroke, dementia, tauopathy diseases, substantial traumatic brain injury, or epilepsy before the index stroke; and (3) the Informant Questionnaire on Cognitive Decline (IQCODE) mean score $<3.4$ obtained within 1 week after stroke onset [16]. Nine of these patients were further excluded due to (1) failure to receive ${ }^{18} \mathrm{~F}$-THK$5351(n=2)$ and ${ }^{18}$ F-florbetapir $(n=3)$ PET scanning, (2) amyloid-positivity on ${ }^{18}$ F-florbetapir PET scanning ( $n$ $=2$ ), (3) recurrent stroke occurring between the index stroke and the study screening procedure $(n=2)$, and (4) persistent moderate to severe dysphasia, which was defined as a score of $>1$ point in the language score of the National Institutes of Health Stroke Scale (NIHSS) [17]. Finally, 63 amyloid-negative patients with recent first-ever stroke were recruited after panel evaluation by neurologists, neuropsychologists, neuroradiologists, and experts in nuclear medicine. There was no significant difference in age, education, stroke volume, and the intervals from stroke onset to screening procedure between patients recruited and excluded.

The study protocol and procedure for obtaining informed consent were complied with the Helsinki Declaration, and were approved by the institutional review board of Chang Gung Memorial Hospital (IRB No. 103$7584 \mathrm{~A}$ and 201601675A0) with the clinical trials registered to Taiwan Food and Drug Administration (1040025953 and 1066015148) and Center of Drug Evaluation (104IND06124 and 106IND03071). All participants provided written informed consent.

\section{Neurocognitive and functional evaluation}

We employed a battery of neurocognitive tests, which had been used in previous studies $[15,18,19]$, to assess a range of cognitive domains around 3 months after stroke onset. The neurocognitive tests in each cognitive domain were summarized in the Sup. Table 1. Of note, the IQCODE test was done twice in the current study; the first test was done within 1 week after stroke onset for pre-stroke cognitive state screening as one of the inclusion criteria, and the second test was done around 3 months after stroke onset for PSCI severity evaluation [20]. Depressive and anxiety symptoms were assessed by the Neuropsychiatric Inventory (NPI) items 4 and 5, respectively. The sequence of tests administration was identical for each individual participant to minimize any possible interference effect between testing. The 
assessment of disability states and cognitive domains mainly consisted of memory, language, executive, and visuospatial functions. Each raw test score was transformed to a $z$ score based on its corresponding normative data. We derived the composite scores for the four cognitive domains (memory, visuospatial, executive, and language functions) by averaging the $z$ scores of the relevant tests. We calculated the overall composite score for general cognitive function by averaging the $z$ scores of all tests that contributed to the four cognitive domains. PSCI was defined as the overall composite score for general cognitive function $<-1[21,22]$.

\section{Imaging evaluation}

\section{Stroke volume evaluation}

Brain CT and MRI were performed at stroke onset to assess acute stroke lesions. The MRI scanning protocol included fluid-attenuated inversion recovery (FLAIR), diffusion-weighted imaging (DWI), and T1-weighted (T1W) sequences. The stroke volume was delineated on the DWI maps for ischemic stroke and on the CT images for hemorrhagic stroke using the PMOD software (version 3.7; PMOD Technologies Ltd., Zurich, Switzerland). We normalized stroke lesion volume according to the head size, which was measured using the Freesurfer software (version 6.0.0).

\section{Brain atrophy, leukoaraiosis, and vascular burden evaluation}

Brain atrophy and leukoaraiosis were evaluated based on the follow-up brain MRI scans performed around 3 months after stroke onset. Axial three-dimensional T1W-MPRAGE (Magnetization Prepared RApid Gradient Echo), susceptibility-weighted imaging (SWI), and FLAIR sequences were acquired on a Siemens 3T MRI system as previously described [15].

We measured the cortical thickness on the T1WMPRAGE images using the Freesurfer software [23]. We evaluated hippocampal atrophy using the Schelten medial temporal lobe atrophy (MTA) score [24, 25]. We measured leukoaraiosis severity on the FLAIR sequence over the periventricular and deep white matter areas for each cerebral hemisphere by the Fazekas scale [26]. Periventricular leukoaraiosis (PVL) was scored as follows: $0=a b-$ sence, 1 = caps or pencil-thin lining, $2=$ smooth halo, or 3 $=$ irregular leukoaraiosis extending into the deep white matter. Further, deep white matter leukoaraiosis (DWML) was scored as follows: $0=$ absence, $1=$ punctuate foci, $2=$ beginning confluence of foci, or $3=$ large confluent areas.

Other imaging biomarkers for small vessel disease were also rated by a senior neuroradiologist. Lobar and deep cerebral microbleeds $(<10 \mathrm{~mm}$ in diameter) were evaluated on SWI [27]. Presence of lacunes (3-15 mm in diameter) and cerebral microbleeds were defined as the presence of one or more lacunes or any cerebral microbleed [28]. Presence of enlarged perivascular space $(<3 \mathrm{~mm}$ in diameter) was counted if there was moderate to severe (grade 2-4) perivascular space in the basal ganglia $[28,29]$.

\section{PET image acquisition and preprocessing procedures}

Both ${ }^{18} \mathrm{~F}$-florbetapir and ${ }^{18} \mathrm{~F}$-THK-5351 positronemission tomography (PET) scans were performed separately using Biograph mMR PET/magnetic resonance (MR) and mCT PET/computed tomography (CT) scanners (Siemens Medical Solutions, Malvern, PA, USA) about 3 months after stroke onset; further, the two scans were conducted at least $48 \mathrm{~h}$ apart to avoid signal interference. A 10-min PET scan of ${ }^{18} \mathrm{~F}$-florbetapir was acquired at $50 \mathrm{~min}$ post-injection of $384 \pm 13 \mathrm{MBq}$ while a 10 -min acquisition of ${ }^{18} \mathrm{~F}$-THK-5351 was performed at $50 \mathrm{~min}$ post-injection of $379 \pm 13 \mathrm{MBq} \cdot{ }^{18} \mathrm{~F}$-florbetapir PET images were reconstructed using point-spread function reconstruction with 2 iterations and 21 subsets, as well as MR-based attenuation correction and scatter and random corrections. Further, ${ }^{18}$ F-THK-5351 PET images were reconstructed using a 3-D ordered subsetsexpectation maximization reconstruction algorithm (4 iterations, 24 subsets; Gaussian filter with $2 \mathrm{~mm}$ full width at half maximum, zoom 3) with CT-based attenuation correction, as well as scatter and random corrections. The final reconstructed images were of $344 \times 344 \times 127$ matrix size $(0.834 \times 834 \times 1.2 \mathrm{~mm}$ voxel size $)$ for ${ }^{18} \mathrm{~F}$ florbetapir and $400 \times 400 \times 148$ matrix size $(0.68 \times 0.68$ $\times 1.5 \mathrm{~mm}$ voxel size) for ${ }^{18} \mathrm{~F}-\mathrm{THK}-5351$.

PET data were motion-corrected, and then spatially normalized into MNI space using MR-based spatial normalization. Image processing was performed using PMOD software (version 3.7; PMOD Technologies Ltd, Zurich, Switzerland) by previously reported protocols [30]. Then, the standardized uptake value ratio (SUVR) image was calculated by using the cerebellar grey matter as the reference region. Amyloid plaque positivity was visually evaluated on ${ }^{18} \mathrm{~F}$-florbetapir PET images [31].

\section{Transformation of ${ }^{18} \mathrm{~F}$-THK-5351 images to Z-Score map}

We applied Z-score analysis to diminish the background signal from the subcortical regions resulting from the intrinsic signal distribution of ${ }^{18} \mathrm{~F}$-THK-5351 images. To calculate the ${ }^{18} \mathrm{~F}$-THK-5351 Z-score image for each subject, a dataset of ${ }^{18} \mathrm{~F}$-THK-5351 PET SUVR images were first obtained from 22 age-matched healthy subjects to establish the ${ }^{18} \mathrm{~F}$-THK5351 signal distribution template as previously described [32-34]. These healthy subjects had (1) age $>55$ years; (2) Mini-Mental State Examination (MMSE) score $\geq 26$ points; (3) no history of stroke, cognitive impairment, parkinsonism, or other neurodegenerative diseases; (4) not taking antiinflammatory medication during the imaging period; and (5) 
negativity for amyloid plaque on ${ }^{18} \mathrm{~F}$-florbetapir PET scanning. These healthy subjects were 65.1 (6.1) years old, with MMSE score of 28.0 (1.1) points. Ten of them were female.

To reduce the spurious uptake and registration error, each spatially normalized ${ }^{18} \mathrm{~F}-\mathrm{THK}-5351$ normal image was smoothed using a 3D Gaussian kernel of $8 \mathrm{~mm}$ full width at half maximum (FWHM). Then two normal ${ }^{18} \mathrm{~F}$ THK-5351 SUVR images of mean $\left(\mu^{\mathrm{TP}}\right)$ and standard deviation $\left(\sigma^{\mathrm{TP}}\right)$ were calculated from the smoothed and spatially normalized SUVR images of the healthy subjects. With voxelwise comparison to the mean and standard deviation values obtained above, the $Z$-score image for each subject with SUVR image $I$ was finally computed as $Z$ $=\frac{I-\mu^{T P}}{\sigma^{T P}}$. Through the transformation, we prominently diminished the background signals in the striatum and thalamus of patients with stroke (Fig. 1). Additionally, we built the ${ }^{18} \mathrm{~F}$-THK-5351 Z-maps for patients with stroke at multiple Z-score thresholds; namely, $Z>2, Z>3, Z>4$, and $Z$ $>5$ (Fig. 2). To investigate the correlation of strokeinduced ${ }^{18} \mathrm{~F}$-THK-5351 uptake with PSCI, we calculated the Z-SUM scores at each Z-map threshold by summing the Z-scores within the whole brain (total Z-SUM scores), ipsilateral cerebral hemisphere (ipsilateral Z-SUM scores), and contralateral cerebral hemisphere (contralateral ZSUM scores). In addition, we also calculated the Z-SUM scores in the stroke core region and the perilesional region.

\section{Statistical analyses}

For descriptive statistics, we performed the two-sample $t$ test, Chi-Square test, and Fisher's exact test for group comparisons. Further, we performed Pearson's correlation analysis to investigate the correlations of the ZSUM scores of ${ }^{18} \mathrm{~F}$-THK-5351 uptake intensity with demographic, vascular, and neurodegenerative factors. Moreover, we analyzed the partial correlations of cognitive performance with Z-SUM scores and other relevant factors after adjusting for age and education covariables.

Since PSCI is associated with multiple factors, we employed multiple regression procedures using the forward selection method to determine the cognitive influence of total Z-SUM scores in three models. In model I, we adopted age and education as confounding factors. In model II, we added vascular factors, including NIHSS, stroke volume, and leukoaraiosis, as explanatory variables. In model III, neurodegenerative factors were further included. We applied 4 thresholds of ${ }^{18} \mathrm{~F}$-THK-5351 Z-score $(Z>2, Z>3, Z>4$, and $Z>5)$ in all the above correlation and regression analyses to determine the most sensitive cutoff level. Subsequently, we applied path analyses to evaluate whether Z-SUM scores of ${ }^{18} \mathrm{~F}$ THK-5351 uptake intensity mediated the associations between stroke volume and PSCI severity after adjusting for age and education and mood conditions from NPI
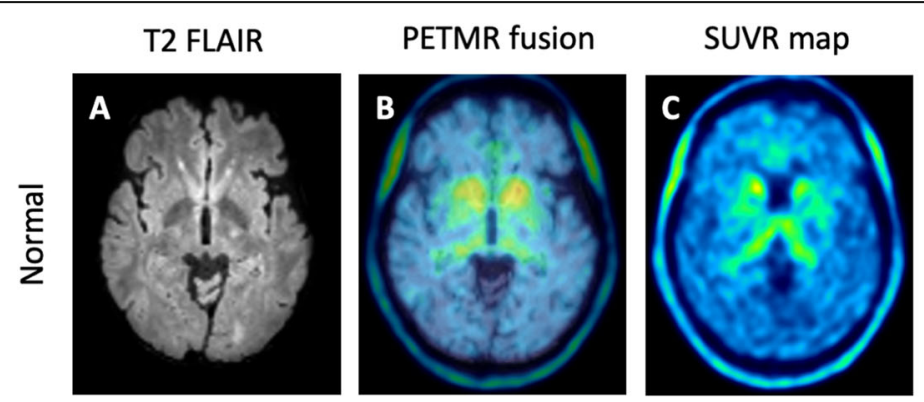

\section{Z-score $>2$}
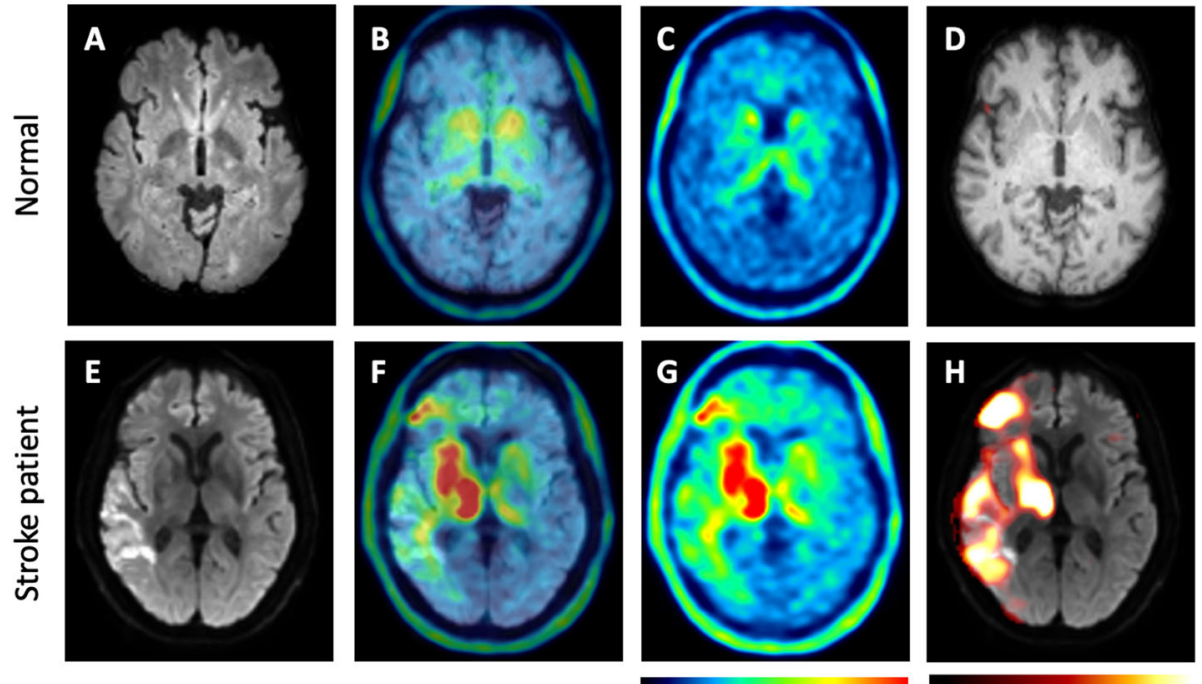

0

5

0 7

Fig. 1 Transforming the ${ }^{18}$ F-THK-5351 SUVR maps to ${ }^{18}$ F-THK-5351 Z-score maps. Regarding a representative healthy subject (a), off-target binding of ${ }^{18} \mathrm{~F}$-THK-5351 to the basal ganglia and thalamus was noted on the SUVR maps (b, c). After transforming SUVR maps to Z-score maps, the signals in the basal ganglia and thalamus were robustly diminished (d). Regarding a patient with right hemisphere ischemic stroke $(\mathbf{e}),{ }^{18} \mathrm{~F}-$ THK-5351 uptake signals were increased around the stroke lesion; further, they were asymmetrically elevated in the basal ganglia and thalamus on SUVR maps $(\mathbf{f}, \mathbf{g})$. On the Z-score map, the ${ }^{18} \mathrm{~F}$-THK-5351 signals on the bilateral basal ganglia and thalamus were markedly suppressed and the stroke-induced ${ }^{18} \mathrm{~F}$-THK-5351 uptake was better visualized (h) 
T2 FLAIR
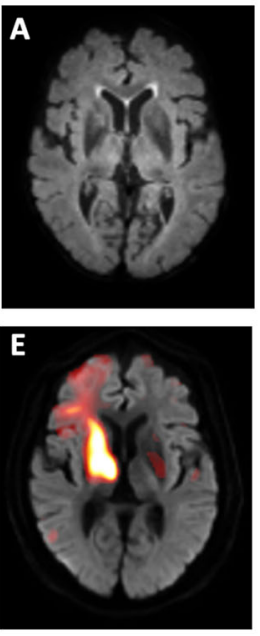

Z-score $>2$
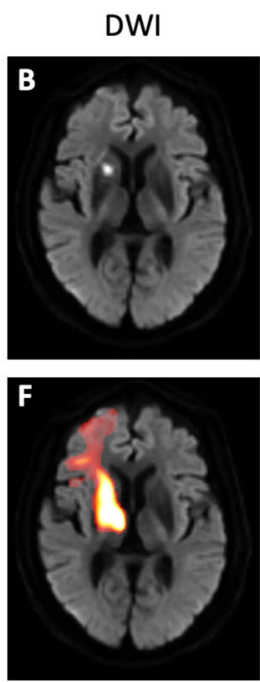

Z-score > 3
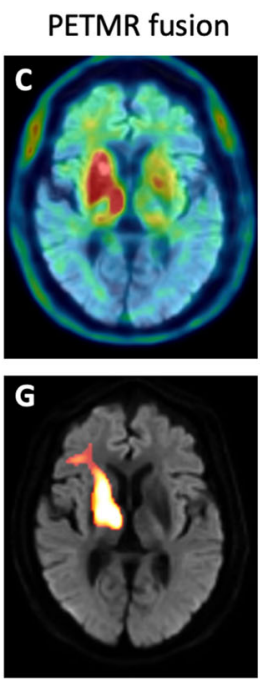

Z-score $>4$
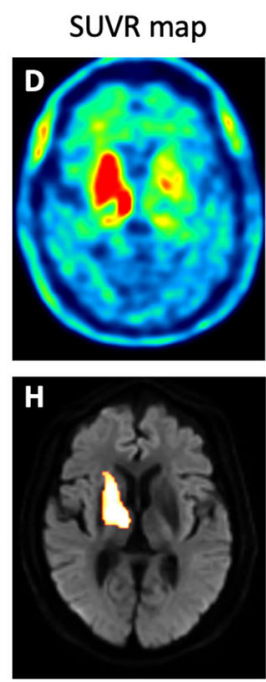

Z-score > 5

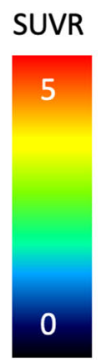

7

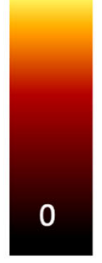

Z-score

Fig. 2 Comparisons of ${ }^{18}$ F-THK-5351 uptake patterns among the SUVR map and Z-maps at multiple Z-score thresholds. A patient with a lacunar infarct at the right internal capsule $(\mathbf{a}, \mathbf{b})$ shows increased ${ }^{18} \mathrm{~F}$-THK-5351 uptake around the stroke lesion on the SUVR maps (c, $\left.\mathbf{d}\right)$. On the Z-maps with sequentially increasing thresholds, the ${ }^{18} \mathrm{~F}-$ THK-5351 uptake regions gradually shrink (e-h). Furthermore, the increased ${ }^{18} \mathrm{~F}-\mathrm{THK}-5351$ uptake might extend to the cerebral cortex without corresponding changes observed on the conventional FLAIR and DWI images

items (Sup. Fig. 1). Statistical analyses were performed with SAS version 9.0 (SAS Institute Inc., New York, USA), and $P$ value $<0.05$ was considered significant.

\section{Results}

We enrolled 63 amyloid-negative, right-handed patients around 3 months after the first-ever index stroke (median 86 days with interquartile range from 73 to 98 days), and 25 of them had PSCI. Patients with PSCI had older age, lower education, and more severe cortical atrophy (Table 1), and also had moderate higher stroke volume, NIHSS, DWML, and MTA scores. Further, there was a moderate to significant difference in the whole brain ${ }^{18} \mathrm{~F}$-THK-5351 uptake intensity between patients with and without PSCI at multiple threshold levels, including total Z-SUM-2 $(Z>2)$, Z-SUM-3 $(Z>$ $3)$, and Z-SUM-4 $(Z>4)$ scores, but not total Z-SUM-5 $(Z>5)$ score. Besides, there was no difference in the presence of enlarged perivascular space, cerebral microbleeds, and lacunes between patients with and without PSCI. In addition, there was no difference in Z-SUM scores in terms of hypertension, diabetes mellitus, dyslipidemia, and current stroke habit for stroke patients and healthy subjects (Sup. Table 2).

\section{Associations of ${ }^{18} \mathrm{~F}$-THK-5351 uptake intensity with stroke features and cognition}

While the total Z-SUM scores of ${ }^{18} \mathrm{~F}$-THK-5351 uptake intensity significantly increased with stroke volume at all thresholds, there was no correlation of total Z-SUM scores with age, education, NIHSS, leukoaraiosis, enlarged perivascular space, cerebral microbleeds, lacunes, brain atrophy factors, and days from stroke onset at most of the Z-score thresholds (Sup. Table 3). The total Z-SUM scores were also significantly correlated with the Montreal Cognitive Assessment (MoCA), Instrumental Activities of Daily Living (IADL), Informant Questionnaire on Cognitive Decline in the Elderly (IQCODE), sum of boxes of Clinical Dementia Rating (CDR-SOB), as well as general cognitive, visuospatial, executive, and language functions, at multiple threshold levels (Table 2). Moreover, there were significant correlations of most cognitive results with other demographic, vascular, and neurodegenerative factors.

${ }^{18} \mathrm{~F}$-THK-5351 Z-SUM scores were further calculated in the stroke core and perilesional regions (Table 3). The Z-SUM scores of stroke core and perilesional regions were both correlated with stroke volume, but not with NIHSS. Furthermore, the cognitive performance was associated with the perilesional Z-SUM scores rather than the stroke core Z-SUM scores after age and education adjustment.

Given the complex associations among demographic data, vascular imaging findings, brain atrophy, and cognition, we built three multiple linear regression models to decipher the influence of ${ }^{18} \mathrm{~F}$-THK-5351 uptake intensity on post-stroke cognitive performance. In model I, age and education were taken as the co-variables (Sup. Table 4). Vascular factors, including NIHSS, stroke volume, and leukoaraiosis, were added as explanatory variables in model II (Sup. Table 5); neurodegenerative factors were further included in model III (Sup. Table 6). Among the 
Table 1 Clinical characteristics of patients with and without post-stroke cognitive impairment

\begin{tabular}{|c|c|c|c|}
\hline \multirow[t]{2}{*}{ Characteristics } & \multicolumn{3}{|l|}{ Mean (SD) } \\
\hline & Without PSCI $(n=38)$ & With PSCI $(n=25)$ & $P$ value \\
\hline Age, year & $61.1(7.1)$ & $68.4(9.4)$ & $<0.01$ \\
\hline Education, year & $10.4(3.1)$ & $7.5(4.6)$ & $<0.01$ \\
\hline Male, No. (\%) & $31(82)$ & $16(64)$ & 0.12 \\
\hline APOE ع4 carrier, No. (\%) & $4(11)$ & $3(12)$ & $1.00^{\mathrm{a}}$ \\
\hline Days between stroke onset and cognition evaluation & $102.4(29.7)$ & $101.6(21.0)$ & 0.91 \\
\hline Days between stroke onset and ${ }^{18} \mathrm{~F}-\mathrm{THK}-5351$ scanning & $92.8(14.8)$ & $104.4(24.4)$ & 0.04 \\
\hline Days between stroke onset and ${ }^{18} \mathrm{~F}$-florbetapir scanning & $100.4(31.6)$ & $101.2(28.4)$ & 0.92 \\
\hline \multicolumn{4}{|l|}{ Common vascular risk factors } \\
\hline Hypertension, No. (\%) & $31(82)$ & $24(96)$ & $0.13^{\mathrm{a}}$ \\
\hline Diabetes mellitus, No. (\%) & $14(37)$ & $6(24)$ & 0.28 \\
\hline Dyslipidemia, No. (\%) & $32(84)$ & $17(68)$ & 0.13 \\
\hline Gout, No. (\%) & $7(18)$ & $3(12)$ & $0.72^{\mathrm{a}}$ \\
\hline PVL score & $0.4(0.8)$ & $0.8(1.1)$ & 0.13 \\
\hline DWML score & $2.6(1.3)$ & $3.2(1.2)$ & 0.08 \\
\hline Enlarged perivascular space, No. (\%) & $11(29)$ & $8(32)$ & 0.80 \\
\hline Lobar microbleed, No. (\%) & $6(16)$ & $6(24)$ & $0.52^{\mathrm{a}}$ \\
\hline Deep microbleed, No. (\%) & $6(16)$ & $2(8)$ & $0.46^{\mathrm{a}}$ \\
\hline Lacune, No. (\%) & $10(26)$ & $4(16)$ & 0.34 \\
\hline \multicolumn{4}{|l|}{ Stroke features } \\
\hline $\mathrm{NIHSS}$ & $1.5(1.3)$ & $2.4(2.3)$ & 0.06 \\
\hline Ischemic stroke, No. (\%) & $35(92)$ & $23(92)$ & $1.00^{\mathrm{a}}$ \\
\hline Left hemisphere stroke, No. (\%) & $17(45)$ & $15(60)$ & 0.24 \\
\hline Supratentorial stroke lesion, No. (\%) & $35(92)$ & $23(92)$ & $1.00^{\mathrm{a}}$ \\
\hline Stroke volume, \% & $3.39 \mathrm{E}-6(4.45 \mathrm{E}-6)$ & $6.18 \mathrm{E}-6(6.57 \mathrm{E}-6)$ & 0.07 \\
\hline MTA score & $0.8(1.0)$ & $1.3(1.3)$ & 0.09 \\
\hline Cortical thickness, mm & $2.44(0.08)$ & $2.37(0.10)$ & 0.01 \\
\hline \multicolumn{4}{|l|}{ Total Z-SUM score at different Z levels } \\
\hline Total Z-SUM-2, Z > 2 & $86763(96363)$ & $136773(126302)$ & 0.09 \\
\hline Total Z-SUM-3, Z > 3 & $43536(58763)$ & $82344(82885)$ & 0.03 \\
\hline Total Z-SUM-4, Z > 4 & $25822(40406)$ & $50558(53591)$ & 0.04 \\
\hline Total Z-SUM-5, Z > 5 & $17404(31432)$ & 31059 (34631) & 0.11 \\
\hline
\end{tabular}

$A P O E \varepsilon 4$ apolipoprotein E \&4, DWML deep white matter leukoaraiosis, MTA medial temporal atrophy, NIHSS National Institutes of Health Stroke Scale, PSCI poststroke cognitive impairment, PVL periventricular leukoaraiosis, Z-SUM sum of ${ }^{18} \mathrm{~F}$-THK-5351 uptake intensity $Z$ scores

Unless otherwise indicated, data are expressed as mean (SD)

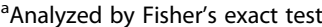

three models, there was a similar trend of associations between total Z-SUM scores of ${ }^{18} \mathrm{~F}$-THK-5351 and cognitive performance, especially for IADL, IQCODE, general cognitive and executive functions, and the total Z-SUM-4 score was most sensitive to cognitive changes (Fig. 3).

\section{Stroke lesion side stratification}

To investigate the influence of stroke lesion side on PSCI, we excluded 5 patients with infratentorial stroke lesions from the analyses. The correlation coefficients of stroke volume with ipsilateral Z-SUM-2 to Z-SUM-5 scores were $0.48,0.54,0.56$, and $0.54(P \mathrm{~s}$ $<0.01$ ), while the correlation coefficients of stroke volume with contralateral Z-SUM-2 to Z-SUM-5 scores were $0.23(P=0.08), 0.26(P=0.04), 0.23(P=$ $0.08)$, and $0.19(P=0.16)$. Moreover, the correlation coefficients between ipsilateral and contralateral hemisphere Z-SUM scores were $0.64(P<0.01), 0.39(P<$ $0.01), 0.13(P=0.35)$, and $0.02(P=0.90)$ for $\mathrm{Z}$ thresholds from 2 to 5 . 
Table 2 Factors associated with cognitive performance

\begin{tabular}{|c|c|c|c|c|c|c|c|c|c|c|c|c|}
\hline & \multicolumn{4}{|c|}{${ }^{18} \mathrm{~F}$-THK-5351 uptake intensity } & \multicolumn{2}{|c|}{$\begin{array}{l}\text { Demographic } \\
\text { factors }\end{array}$} & \multicolumn{4}{|c|}{ Vascular factors } & \multicolumn{2}{|c|}{$\begin{array}{l}\text { Neurodegenerative } \\
\text { factors }\end{array}$} \\
\hline & $\begin{array}{l}\text { Total Z- } \\
\text { SUM-2 }\end{array}$ & $\begin{array}{l}\text { Total Z- } \\
\text { SUM-3 }\end{array}$ & $\begin{array}{l}\text { Total Z- } \\
\text { SUM-4 }\end{array}$ & $\begin{array}{l}\text { Total Z- } \\
\text { SUM-5 }\end{array}$ & $\begin{array}{l}\text { Age, } \\
\text { year }\end{array}$ & $\begin{array}{l}\text { Education, } \\
\text { year }\end{array}$ & NIHSS & $\begin{array}{l}\text { Stroke } \\
\text { volume, } \\
\%\end{array}$ & $\begin{array}{l}\text { PVL } \\
\text { score }\end{array}$ & $\begin{array}{l}\text { DWML } \\
\text { score }\end{array}$ & $\begin{array}{l}\text { MTA } \\
\text { score }\end{array}$ & $\begin{array}{l}\text { Cortical } \\
\text { thickness, } \\
\text { mm }\end{array}$ \\
\hline $\mathrm{MoCA}$ & $-0.26^{*}$ & $-0.29^{*}$ & $-0.28^{*}$ & -0.22 & $-\overline{0.42^{* * *}}$ & $0.59 * * *$ & $-\overline{0.55^{* * *}+}$ & $-0.23 \dagger$ & -0.23 & $-0.32^{*}+$ & -0.11 & $0.55^{* * *} \dagger$ \\
\hline NPI & 0.21 & 0.21 & 0.21 & 0.17 & 0.08 & -0.16 & $0.58^{* * *}+$ & $0.28^{*}+$ & 0.06 & 0.19 & -0.10 & $-0.32 \dagger$ \\
\hline $\begin{array}{l}\text { Depressive } \\
\text { symptoms }^{a}\end{array}$ & 0.07 & 0.05 & 0.04 & 0.01 & 0.06 & -0.11 & $0.62^{* * *} \dagger$ & 0.19 & 0.25 & 0.22 & -0.06 & -0.24 \\
\hline Anxiety $^{b}$ & -0.10 & -0.13 & -0.13 & -0.13 & 0.12 & $-0.31^{*}$ & $0.28^{*}$ & -0.10 & 0.13 & -0.03 & 0.13 & -0.20 \\
\hline IADL & $0.37^{* *}+$ & $0.41^{* * *} \dagger$ & $0.39^{* *}+$ & $0.31^{*}+$ & $0.31^{*}$ & $-0.47^{* * *}$ & $0.58^{* * *}+$ & $0.29^{*}+$ & 0.12 & 0.19 & 0.03 & $-0.44^{* * *}$ \\
\hline $\mathrm{IQCODE}^{\mathrm{C}}$ & $0.42^{* * *} \dagger$ & $0.42^{* * *} \dagger$ & $0.37^{* *}+$ & $0.29^{*}+$ & $0.34^{* *}$ & -0.25 & $0.42^{* * *}+$ & $0.4^{* *}+$ & 0.24 & $0.43^{* * *} \dagger$ & 0.19 & $-0.35^{* *}$ \\
\hline CDR-SOB & $0.31^{*}$ & $0.36^{* *}+$ & $0.35^{* *}+$ & $0.27^{*}+$ & $0.35^{* *}$ & $-0.32^{* *}$ & $0.57^{* * *}+$ & $0.29^{*}+$ & 0.18 & 0.23 & 0.03 & $-0.45^{* * *} \dagger$ \\
\hline \multicolumn{13}{|c|}{ Composite cognitive $z$ score } \\
\hline $\begin{array}{l}\text { General } \\
\text { cognitive } \\
\text { function }\end{array}$ & $-0.28^{*}$ & $-\overline{0.36^{* *+}+}$ & $-\overline{0.38^{* *}+}$ & $-\overline{0.34^{* *} \dagger}$ & $-\overline{0.26^{*}}$ & $0.51^{* * *}$ & - & $-0.31^{*} \dagger$ & -0.24 & $-0.29 * \dagger$ & -0.15 & $0.39^{* *}$ \\
\hline $\begin{array}{l}\text { Memory } \\
\text { function }\end{array}$ & -0.11 & -0.16 & -0.18 & -0.15 & $-\overline{0.35 * *}$ & $0.36^{* *}$ & - & -0.14 & $-\overline{0.38^{* *} \dagger}$ & $-0.27^{*}$ & -0.18 & $0.36^{* *}$ \\
\hline $\begin{array}{l}\text { Visuospatial } \\
\text { function }\end{array}$ & $-0.28^{*}$ & $-0.28^{*}$ & $-0.27^{*}$ & -0.23 & $-\overline{0.30^{*}}$ & 0.21 & -0.25 & $-0.24 \dagger$ & -0.09 & $-0.26^{*}$ & $0.12+$ & $0.26^{*}$ \\
\hline $\begin{array}{l}\text { Executive } \\
\text { function }\end{array}$ & $-\overline{0.34^{* *+}+}$ & $\overline{-} .44^{* * *} \dagger$ & $-\overline{0.45^{* * *} \dagger}$ & $-\overline{0.43^{* * *+} \dagger}$ & -0.09 & $0.39 * *$ & $-\overline{0.41^{* * *}+}$ & $-0.36^{* *}+$ & -0.06 & -0.21 & -0.10 & $0.32^{* *}$ \\
\hline $\begin{array}{l}\text { Language } \\
\text { function }\end{array}$ & -0.23 & $-0.29^{*} \dagger$ & $-0.32^{*} \dagger$ & $-0.31^{*}+$ & -0.12 & $0.66^{* * *}$ & -0.25 & $-0.28^{*} \dagger$ & -0.09 & $-0.22+$ & -0.14 & 0.24 \\
\hline
\end{tabular}

CDR clinical dementia rating, DWML deep white matter leukoaraiosis, IADL instrumental activities of daily living, IQCODE informant questionnaire on cognitive decline in the elderly, MoCA Montreal cognitive assessment, MTA medial temporal atrophy, NIHSS National Institutes of Health Stroke Scale, NPI neuropsychiatric inventory, PVL periventricular leukoaraiosis, SOB sum of boxes, Z-SUM sum of ${ }^{18} \mathrm{~F}-\mathrm{THK}-5351$ uptake intensity $Z$ scores

${ }^{*} P<0.05 ;{ }^{* *} P<0.01 ;{ }^{* * *} P<0.001 ;+P<0.05$ after adjustment for age and education

${ }^{a}$ Evaluated by the NPI depression item 4

${ }^{\mathrm{b}}$ Evaluated by the NPI anxiety item 5

${ }^{C}$ Performed around 3 months after stroke

We further stratified patients into left- and righthemispheric stroke. There was no significant difference in the demographic data, imaging findings, and cognitive results between patients with right- and left-hemispheric stroke (Sup. Table 7). The total Z-SUM scores were significantly correlated with CDR-SOB, general cognitive function, and each cognitive domain function in patients with left-hemispheric stroke and with executive function in patients with right-hemispheric stroke after adjustment for age and education. Furthermore, cognitive performance was more prominently correlated with the ipsilateral Z-SUM scores (Table 4), but not with the contralateral Z-SUM scores (Sup. Table 8). With regard to stroke volume, it was correlated with general cognitive and language functions in patients with lefthemispheric stroke, and with IQCODE, CDR-SOB, and general cognitive and executive functions in patients with right-hemispheric stroke.

We conducted path analyses to explore the mediation effects of Z-SUM scores on the associations between stroke volume and PSCI severity after adjusting for age, education, and depressive symptoms (Fig. 4) and anxiety (Sup. Fig. 2) covariables (Sup. Table 9). We adopted the Z-SUM-4 score as the mediator because it was most sensitive to cognitive changes based on the above regression model results. We selected cognitive tests both correlated with stroke volume and Z-SUM-4 scores as the endogenous variables. Path analyses showed that stroke volume had significant direct effects on the total and ipsilateral Z-SUM-4 scores in patients with either left or right hemisphere stroke. In addition to the direct cognitive influence from stroke volume, ipsilateral Z-SUM-4 scores also significantly mediated the associations between stroke volume and language function in patients with left-hemispheric stroke and between stroke volume and executive function in patients with righthemispheric stroke, respectively.

\section{Discussion}

PSCI is usually presented in patients 3 to 6 months after stroke onset. A previous study suggested that neuroinflammation plays a role in the modulation of post-stroke 
Table 3 Correlations of Z-SUM scores of the stroke core and perilesional regions with stroke severity, stroke volume, and cognitive performance

\begin{tabular}{|c|c|c|c|c|c|c|c|c|}
\hline & \multicolumn{4}{|c|}{ Stroke core regions } & \multirow[b]{2}{*}{ Z-SUM-2 } & \multicolumn{3}{|c|}{ Perilesional regions } \\
\hline & Z-SUM-2 & Z-SUM-3 & Z-SUM-4 & Z-SUM-5 & & Z-SUM-3 & Z-SUM-4 & Z-SUM-5 \\
\hline NIHSS & 0.18 & 0.17 & 0.14 & 0.10 & 0.22 & 0.24 & 0.19 & 0.14 \\
\hline Stroke volume, \% & $0.73^{* * *}+$ & $0.69^{* * *} \dagger$ & $0.63^{* * *} \dagger$ & $0.56^{* * *} \dagger$ & $0.41^{* * *} \dagger$ & $0.51^{* * *}+$ & $0.55^{* * *} \dagger$ & $0.54^{* * *} \dagger$ \\
\hline MoCA & -0.13 & -0.12 & -0.09 & -0.05 & $-0.26^{*}$ & $-0.29^{*}$ & $-0.29^{*}$ & -0.24 \\
\hline NPI & 0.14 & 0.12 & 0.09 & 0.05 & 0.20 & 0.21 & 0.22 & 0.19 \\
\hline Depressive symptoms ${ }^{a}$ & 0.05 & 0.04 & 0.00 & -0.04 & 0.07 & 0.05 & 0.04 & 0.02 \\
\hline Anxiety $^{\mathrm{b}}$ & -0.10 & -0.10 & -0.10 & -0.10 & -0.09 & -0.13 & -0.13 & -0.13 \\
\hline IADL & 0.19 & 0.18 & 0.15 & 0.12 & $0.37^{* *}+$ & $0.41^{* * *} \dagger$ & $0.41^{* *}+$ & $0.33^{* *}+$ \\
\hline $\mathrm{IQCODE}^{\mathrm{C}}$ & 0.14 & 0.11 & 0.07 & 0.02 & $0.42^{* * *} \dagger$ & $0.44^{* * *}+$ & $0.40^{* *} \dagger$ & $0.33^{* *} \dagger$ \\
\hline CDR-SOB & 0.15 & 0.13 & 0.11 & 0.08 & $0.31^{*}$ & $0.37^{* *}+$ & $0.37^{* *+}$ & $0.29^{*}+$ \\
\hline \multicolumn{9}{|l|}{ Composite cognitive $z$ score } \\
\hline General cognitive function & $-0.26^{*}$ & $-0.25^{*}$ & -0.23 & -0.21 & $-0.27^{*}$ & $-0.35^{* *}+$ & $-0.38^{* *}+$ & $-0.35^{* *} \dagger$ \\
\hline Memory function & -0.12 & -0.11 & -0.10 & -0.09 & -0.11 & -0.16 & -0.18 & -0.15 \\
\hline Visuospatial function & -0.18 & -0.18 & -0.17 & -0.16 & $-0.28^{*}$ & $-0.28^{*}$ & $-0.27^{*}$ & -0.23 \\
\hline Executive function & $-0.28^{*}$ & $-0.27^{*}$ & -0.25 & -0.21 & $-0.33^{* *}+$ & $-0.43^{* * *}+$ & $-0.46^{* * *} \dagger$ & $-0.44^{* * *} \dagger$ \\
\hline Language function & $-0.31^{*}$ & $-0.31^{*}$ & $-0.30^{*}$ & $-0.27^{*}$ & -0.22 & $-0.28^{*}+$ & $-0.31^{*}+$ & $-0.30^{*} \dagger$ \\
\hline
\end{tabular}

$C D R$ clinical dementia rating, IADL instrumental activities of daily living, IQCODE informant questionnaire on cognitive decline in the elderly, MoCA Montreal cognitive assessment, NIHSS National Institutes of Health Stroke Scale, NPI neuropsychiatric inventory, SOB sum of boxes, Z-SUM sum of ${ }^{18} \mathrm{~F}$-THK-5351 uptake intensity $Z$ scores

${ }^{*} P<0.05 ;{ }^{*} P<0.01 ;{ }^{* * *} P<0.001 ;+P<0.05$ after adjustment for age and education

${ }^{\mathrm{a} E v a l u a t e d}$ by the NPI depression item 4

${ }^{\mathrm{b}}$ Evaluated by the NPI anxiety item 5

'Performed around 3 months after stroke
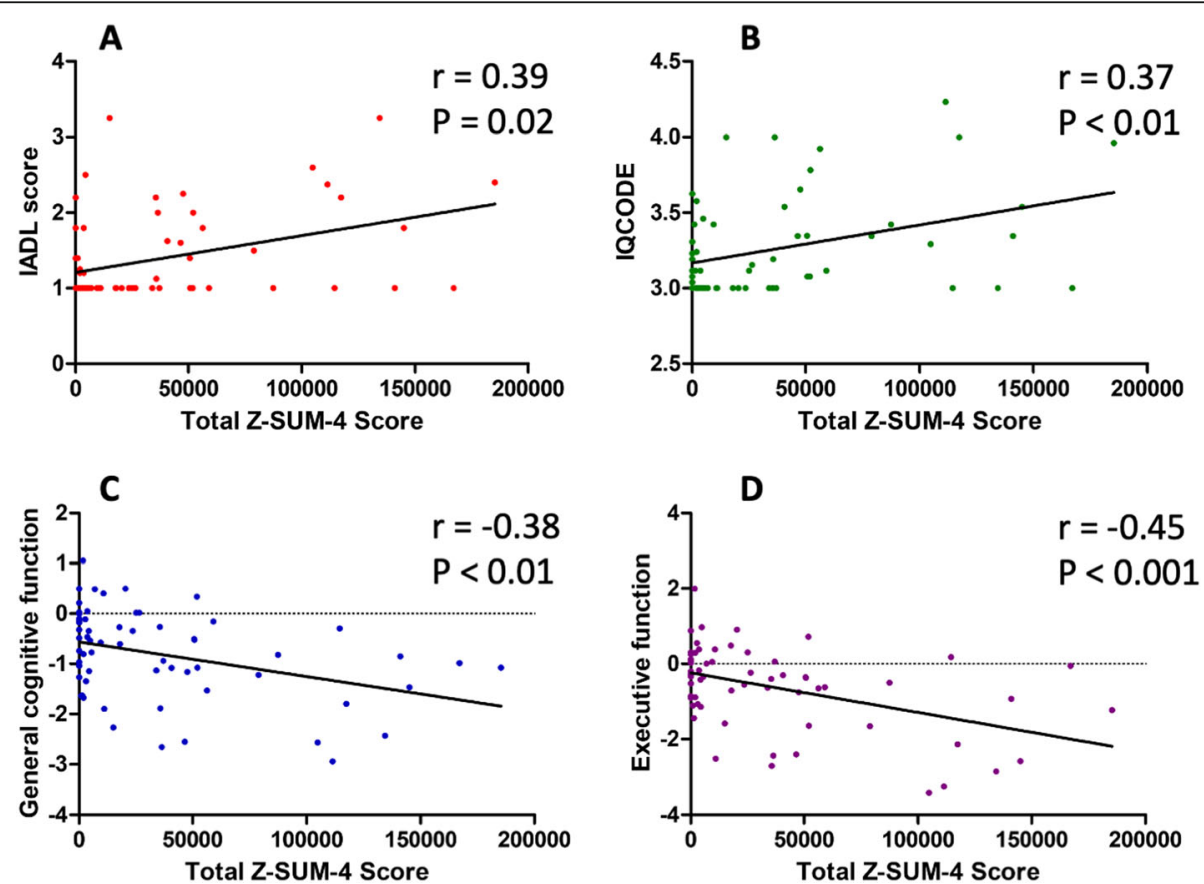

Fig. 3 The correlations of cognitive results with total Z-SUM-4 scores, the normalized ${ }^{18} \mathrm{~F}-\mathrm{THK}$ - 5351 uptake intensity. The total Z-SUM-4 score was significantly correlated with Instrumental Activities of Daily Living (IADL) score (a), Informant Questionnaire on Cognitive Decline in the Elderly (IQCODE) score (b), general cognitive function (c), and executive function performance (d) 
Table 4 Correlations of Z-SUM scores with cognitive performance in patients with left and right hemisphere stroke

\begin{tabular}{|c|c|c|c|c|c|c|c|c|c|}
\hline & MoCA & IADL & IQCODE $^{\mathrm{a}}$ & $\begin{array}{l}\text { CDR- } \\
\text { SOB }\end{array}$ & $\begin{array}{l}\text { General cognitive } \\
\text { function }\end{array}$ & Memory & $\begin{array}{l}\text { Visuospatial } \\
\text { function }\end{array}$ & $\begin{array}{l}\text { Executive } \\
\text { function }\end{array}$ & Language \\
\hline \multicolumn{10}{|c|}{ Patients with left hemisphere stroke $(n=30)$} \\
\hline $\begin{array}{l}\text { Stroke volume, } \\
\%\end{array}$ & -0.24 & 0.25 & 0.33 & 0.13 & $-0.36 \dagger$ & -0.15 & -0.19 & -0.34 & $-0.56^{*}$ \\
\hline Total Z-SUM-2 & $-0.41^{*}$ & $0.46^{*}$ & $0.40^{*}$ & $0.45^{*}+$ & $-0.48^{*}+$ & $-0.38^{*}$ & $-0.41^{*}+$ & $-0.40^{*}$ & $-0.42^{*}$ \\
\hline Total Z-SUM-3 & $-\overline{0.44^{*}+}$ & $0.48^{*} \dagger$ & 0.34 & $0.49^{*}+$ & $-0.54^{*}+$ & $-0.39 *+$ & $-0.36+$ & $-0.49^{*}+$ & $-0.51^{*}+$ \\
\hline Total Z-SUM-4 & $-\overline{0.41^{*}+}$ & $0.41^{*}$ & 0.24 & $0.46^{*}+$ & $-0.52^{*} \dagger$ & $-0.36+$ & $-0.30 \dagger$ & $-0.48^{*} \dagger$ & $-0.52^{*} \dagger$ \\
\hline Total Z-SUM-5 & $-0.32 \dagger$ & 0.32 & 0.16 & $0.36^{*} \dagger$ & $-0.44^{*}+$ & $-0.29+$ & -0.20 & $-0.43^{*}+$ & $-0.48^{*}+$ \\
\hline $\begin{array}{l}\text { Ipsilateral Z- } \\
\text { SUM-2 }\end{array}$ & $\begin{array}{l}- \\
0.57^{*}+\end{array}$ & $0.55^{*}+$ & $0.41^{*}+$ & $0.55^{*}+$ & $-0.57^{*}+$ & $-0.42^{*} \dagger$ & $-0.46^{*}+$ & $-0.50 \dagger$ & $-0.52 \dagger$ \\
\hline $\begin{array}{l}\text { Ipsilateral Z- } \\
\text { SUM-3 }\end{array}$ & $\begin{array}{l}- \\
0.56^{*}+\end{array}$ & $0.54^{*}+$ & 0.34 & $0.55^{*}+$ & $-0.59^{*}+$ & $-0.40^{*} \dagger$ & $-0.40^{*}+$ & $-0.55^{*}+$ & $-0.56^{*}+$ \\
\hline $\begin{array}{l}\text { Ipsilateral Z- } \\
\text { SUM-4 }\end{array}$ & $-\overline{0.51^{*+}+}$ & $0.47^{*}+$ & 0.25 & $0.50^{*}+$ & $-0.55^{*}+$ & $-0.36+$ & $-0.32+$ & $-0.53^{*}+$ & $-0.56^{*}+$ \\
\hline $\begin{array}{l}\text { Ipsilateral Z- } \\
\text { SUM-5 }\end{array}$ & $-\overline{0.40^{*}+}$ & $0.37^{*}+$ & 0.15 & $0.39^{*}+$ & $-0.47^{*}+$ & $-0.28+$ & $-0.22 \dagger$ & $-0.47^{*} \dagger$ & $-0.52^{*} \dagger$ \\
\hline \multicolumn{10}{|c|}{ Patients with Right Hemisphere Stroke ( $n=28$ ) } \\
\hline $\begin{array}{l}\text { Stroke volume, } \\
\%\end{array}$ & -0.27 & 0.30 & $0.43^{*}+$ & $0.41^{*}+$ & $-0.33+$ & -0.20 & -0.37 & $-0.44^{*}+$ & 0.01 \\
\hline Total Z-SUM-2 & -0.14 & 0.23 & $0.39^{*}$ & 0.20 & -0.09 & 0.08 & -0.17 & -0.27 & -0.05 \\
\hline Total Z-SUM-3 & -0.15 & 0.29 & $0.45^{*}$ & 0.25 & -0.19 & 0.01 & -0.20 & $-0.38^{*}+$ & -0.07 \\
\hline Total Z-SUM-4 & -0.13 & 0.32 & $0.46^{*}$ & 0.26 & -0.22 & -0.01 & -0.24 & $-0.42^{*} \dagger$ & -0.08 \\
\hline Total Z-SUM-5 & -0.07 & 0.26 & $0.38^{*}$ & 0.18 & -0.21 & 0.00 & -0.28 & $-0.41^{*} \dagger$ & -0.07 \\
\hline $\begin{array}{l}\text { Ipsilateral Z- } \\
\text { SUM-2 }\end{array}$ & -0.16 & 0.28 & $0.43^{*}$ & 0.19 & -0.16 & 0.06 & -0.25 & $-0.37 \dagger$ & -0.08 \\
\hline $\begin{array}{l}\text { Ipsilateral Z- } \\
\text { SUM-3 }\end{array}$ & -0.16 & 0.32 & $0.47^{*}$ & 0.24 & -0.22 & 0.00 & -0.25 & $-0.44^{*} \dagger$ & -0.09 \\
\hline $\begin{array}{l}\text { Ipsilateral Z- } \\
\text { SUM-4 }\end{array}$ & -0.13 & 0.33 & $0.46^{*}$ & 0.26 & -0.23 & -0.02 & -0.25 & $-0.44^{*} \dagger$ & -0.08 \\
\hline $\begin{array}{l}\text { Ipsilateral Z- } \\
\text { SUM-5 }\end{array}$ & -0.07 & 0.26 & $0.39^{*}$ & 0.18 & -0.21 & 0.00 & -0.28 & $-0.41^{*}+$ & -0.07 \\
\hline
\end{tabular}

$C D R$ clinical dementia rating, IADL instrumental activities of daily living, IQCODE informant questionnaire on cognitive decline in the elderly, MoCA Montreal cognitive assessment, NIHSS National Institutes of Health Stroke Scale, NPI neuropsychiatric inventory, SOB sum of boxes, Z-SUM sum of ${ }^{18} \mathrm{~F}$-THK-5351 uptake intensity Z scores

${ }^{*} P<0.05 ; \dagger P<0.05$ after adjustment for age and education

aperformed around 3 months after stroke

cognitive performance [35]. In this study, we built an ${ }^{18}$ F-THK-5351 PET imaging template using data obtained from amyloid-negative healthy subjects to diminish background signals in the basal ganglia, thalamus, and brainstem. We enrolled amyloid-negative patients with first-ever stroke to investigate the RA extent, which was defined as the Z-SUM scores on ${ }^{18} \mathrm{~F}$-THK-5351 PET imaging. Patients with PSCI had higher total ZSUM scores of ${ }^{18} \mathrm{~F}$-THK-5351 uptake intensity than those without PSCI. The total and ipsilateral Z-SUM scores were also associated with the patterns of poststroke cognitive performance. Furthermore, path analyses indicated that the influence of stroke events on PSCI resulted from stroke volume itself as well as the
RA extent indicated as the Z-SUM scores. This is the first study to demonstrate the associations between PSCI and RA extent on ${ }^{18} \mathrm{~F}$-THK-5351 PET imaging.

We observed increased ${ }^{18} \mathrm{~F}$-THK-5351 uptake intensity on Z-maps around the stroke lesion, which faded with distance away from the lesion core. This uptake distribution pattern could mimic the scar-like structures resulting from the proliferation and migration of reactive astrocytes in the ischemic penumbra [36, 37]. These scar-forming astrocytes have been reported to separate nonfunctional, nonneural lesion core tissue from immediately surrounding and potentially functional neural tissue [13]. Although astrocytes may produce scaffolding proteins to guide neuron growth [38], astrocyte scars 

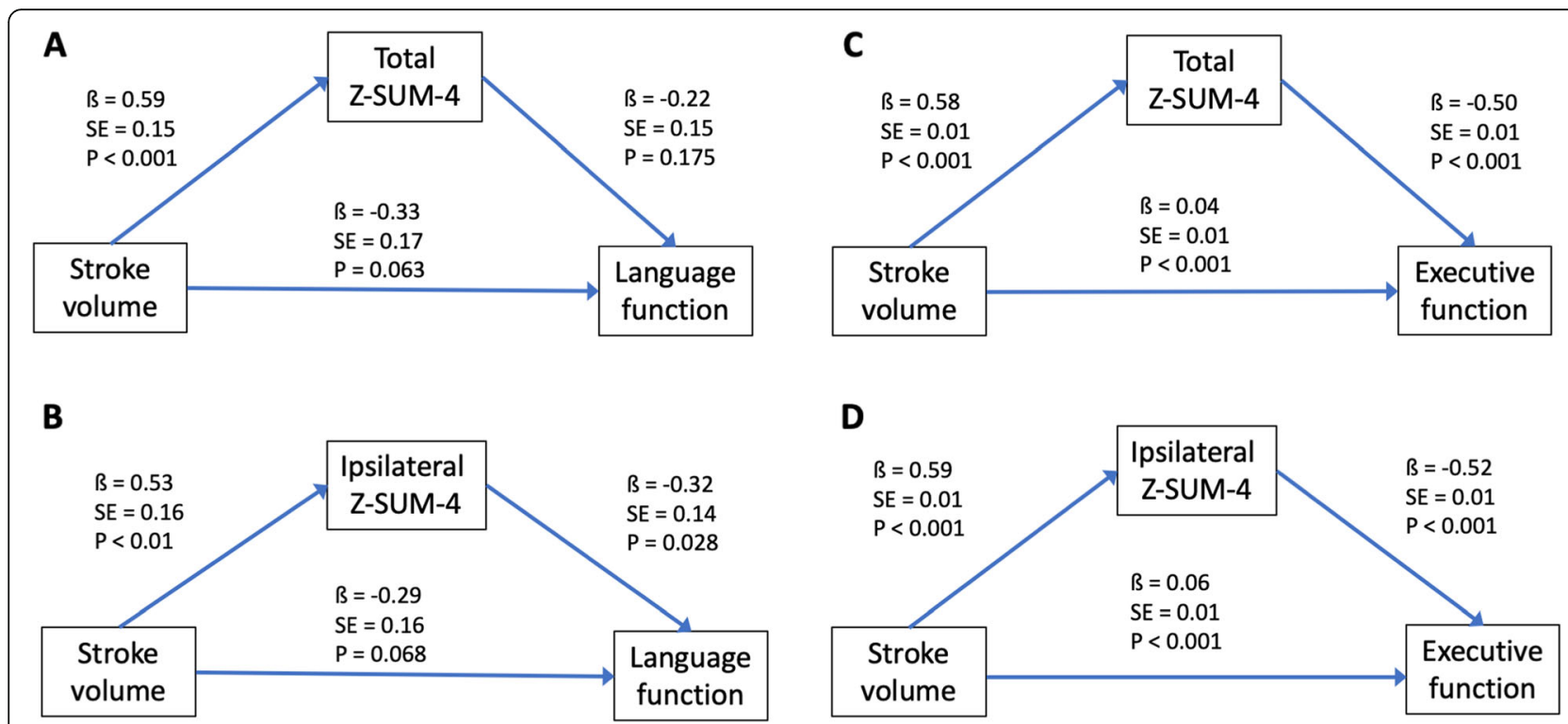

Fig. 4 Path analyses of stroke effects on cognition via total or ipsilateral Z-SUM-4 scores after age, education and depressive symptoms adjustment. Stroke volume contributes significantly to cognitive function as well as total and ipsilateral Z-SUM-4 scores in patients with either lefthemispheric $(\mathbf{a}, \mathbf{b})$ or right-hemispheric $(\mathbf{c}, \mathbf{d})$ stroke. Further, ipsilateral Z-SUM-4 scores can partly mediate language influence from stroke volume in patients with left-hemispheric stroke (b). Likewise, total and ipsilateral Z-SUM-4 scores can partly mediate executive influence from stroke volume in patients with right-hemispheric stroke $(\mathbf{c}, \mathbf{d})$

have been widely regarded as a major impediment to axon regeneration after CNS injury [39]. In addition, disrupted glioneuronal interaction can cause synaptic dysfunction and cognitive impairment [40].

The cognitive influence from stroke lesions not only results from the focal factors, such as stroke volume and location, but also can be attributed to the perilesional and remote effects of these lesions [41]. Previous diffusion tensor imaging studies have reported that changes in perilesional and remote areas had a greater influence on cognitive manifestations than the stroke lesion core itself $[42,43]$. Similarly in our study, the perilesional ZSUM scores rather than the stroke core Z-SUM scores were associated with cognitive performance. In addition, we also stratified the Z-SUM scores into total, ipsilateral, and contralateral scores, and we found ipsilateral ZSUM scores tended to have more correlation with cognitive performance than total Z-SUM scores. Future multi-modality imaging studies could employ in vivo RA visualization on PET images to investigate the neuroinflammation influence on perilesional and remote areas via structural and functional connectivity analyses, which might help understand the pathophysiological bases of PSCI [41].

In our study, we applied the Z-map method to contrast the stroke-induced RA extent on ${ }^{18} \mathrm{~F}$-THK-5351 PET imaging, which may mimic the inflammatory penumbra $[44,45]$. As inflammation is a common reaction to miscellaneous physical conditions, applying a threshold to acquire the Z-SUM scores would help to filter out signals not induced by stroke lesion. We found the Z-SUM-4 scores (with values at least 4 standard deviations higher than the mean at each voxel) were more sensitive to PSCI severity.

Post-stroke neuroinflammation is a diffuse process. In our study, we explored the associations of bilateral hemisphere RA with stroke volume and cognition. We found that stroke volume was significantly correlated with ipsilateral Z-SUM scores, but its correlations with contralateral Z-SUM scores were relatively minor at multiple $Z$ thresholds $(Z>2, Z>3$, and $Z>4)$. Further, the correlations between bilateral cerebral hemispheric Z-SUM-2 and Z-SUM-3 scores were significant. Besides, we also found the contralateral Z-SUM scores of stroke patients were higher than the average Z-SUM scores of bilateral cerebral hemispheres of the 22 healthy controls (at $Z>$ 2, $Z>3$, and $Z>4$ thresholds; Sup. Table 10). However, the Z-SUM scores in the contralateral hemisphere were not associated with cognitive performance. These findings suggest that post-stroke neuroinflammation is a diffuse process, which is more prominent in the ipsilateral hemisphere and minor in the contralateral hemisphere.

${ }^{18} \mathrm{~F}$-THK-5351 has the alternative affinity for monoamine oxidase- $\mathrm{B}$ (MAO-B), and MAO-B is largely expressed in astrocytes in response to CNS damage [12, 13]. We took the advantage of such characteristics to explore RA presentations in first-ever stroke patients. In our study, we adopted several major steps to decrease 
the possibility of cross-binding of ${ }^{18} \mathrm{~F}$-THK-5351 to tau protein. Firstly, we included amyloid-negative first-ever stroke patients to decrease the possibility of co-existing AD. Secondly, we recruited stroke patients without medical history and imaging results suggestive of tauopathy, including $\mathrm{AD}$, progressive supranuclear palsy, corticobasal degeneration, and fronto-temporal dementia, and the patient recruitment was evaluated after consensus by a multidiscipline team. Furthermore, as tau protein has been reported as a marker of axonal injury, ischemic stroke may induce a transient tau protein increase in human CSF, with a peak 1 week after stroke onset and a normalization after 3 months $[46,47]$. In our study, we recruited patients around 3 months after stroke to decrease the acute stroke-related effect on tau protein formation. Although the transformed ${ }^{18} \mathrm{~F}-\mathrm{THK}-5351$ uptake values may be more relevant to stroke-induced RA and appear correlated with PSCI after these procedures, the actual accuracy and sensitivity of ${ }^{18} \mathrm{~F}$-THK5351 to RA in stroke patients need to be clarified in future studies. Further pathological and neuroimaging investigations could provide more direct evidence on the interaction between stroke-induced RA and PSCI.

Both acute ischemic stroke and hemorrhagic stroke are associated with glial toxicity and cell injury, but with different spatio-temporal neuroinflammatory processes and mechanisms $[48,49]$. Although astrocytes have differential roles in the recovery patterns of ischemic and hemorrhagic stroke, the long-term GFAP-positive astrocytic plasticity could be similar after both ischemic and hemorrhagic stroke $[50,51]$. In our sub-analyses, the associations between Z-SUM scores and PSCI presentations were significant in ischemic patients, and the trends were similar to the results of the pooled population (Sup. Table 11). The sample size of patients with hemorrhagic stroke $(n=5)$ was insufficient to investigate such associations. As there was no difference in clinical characteristics, stroke volume, and Z-SUM scores between patients with ischemic and hemorrhagic stroke, we pooled these two types of patients together to explore the influence of RA on PSCI (Sup. Table 12). However, RA response to ischemic and hemorrhagic stroke may be dynamic and versatile in the recovery process, and further longitudinal studies are warranted to investigate the RA influence on PSCI presentations in different stroke subtypes.

Patients with stroke are more susceptible to attention, spatial ability, language, and executive function impairments rather than memory problem [21]. These findings were in line with our study result that stroke volume and total Z-SUM scores of ${ }^{18} \mathrm{~F}$-THK-5351 were correlated with most of cognitive results, but not with memory performance (Table 2). Education attainment is associated with PSCI severity, and we have taken education into account when investigating the associations between RA extent and PSCI performance in the correlation, regression, and path analyses. Other risk factors, such as age, stroke volume, as well as hippocampal and cortical atrophy, are associated with the incidence of PSCI $[1-4,52]$. Similar findings were also noted in our study. After adjusting for confounding factors in the multiple regression analyses, the total Z-SUM scores were still correlated with IADL, IQCODE, and the general cognitive and executive functions in most models.

Both PSCI and post-stroke depression are associated with late worsening of disability, and an immunological hypothesis is one of the mechanisms of these two stroke sequelae [51]. Besides, post-stroke depression could also be a potential source of PSCI. In our study, there was no difference in depressive symptoms and anxiety between patients with and without PSCI, and the influence of RA extent on cognitive performance remained significant after adjusting for these mood conditions in path analyses. As the etiology of post-stroke depression remains controversial, further studies are necessary to investigate the associations between neuroimaging and fluid inflammatory biomarkers associated with post-stroke depression [53].

Left-hemispheric stroke is reported as an important risk factor for PSCI [54]. Similar findings were noted in our study that patients with left hemisphere stroke tended to score lower in each cognitive domain battery than patients with right hemisphere stroke (Sup. Table 7). When focusing on the cognitive influence of stroke-induced RA on specific cerebral hemisphere, ipsilateral Z-SUM scores were correlated with most cognitive results in patients with left hemisphere stroke, while ipsilateral Z-SUM scores were correlated with IQCODE and executive function in patients with right hemisphere stroke (Table 4). Furthermore, under path analyses, PSCI severity could be attributed to stroke lesion volume directly as well as stroke-induced inflammation indirectly in patients with right or left hemisphere stroke, suggesting that strokeinduced RA may have a modulating effect on PSCI occurrence (Sup. Table 9).

Currently, other radioligands targeting the neuroinflammatory process are under development. Microglia can be imaged using the ${ }^{11} \mathrm{C}$-PK11195 and ${ }^{11} \mathrm{C}$-PBR28 radiotracers, which have a high affinity for the $18-\mathrm{kDa}$ translocator protein found in the mitochondria of microglia. Post-stroke microglia activation has been investigated using ${ }^{11} \mathrm{C}$-PK11195 PET imaging, but there is limited literature on the relationship between ${ }^{11} \mathrm{C}$ PK11195 findings and PSCI $[9,55]$. In contrast, the ${ }^{11} \mathrm{C}$ deuterium-L-deprenyl ( $\left.{ }^{11} \mathrm{C}-\mathrm{DED}\right)$ and ${ }^{18} \mathrm{~F}-\mathrm{SMBT}-1$ radiotracers are designed to bind to $\mathrm{MAO}-\mathrm{B}$ in astrocytes [5]. The utility of ${ }^{11} \mathrm{C}$-DED radiotracer is limited by the short half-life of carbon-11 and their suboptimal 
binding specificity [56]. The ${ }^{18} \mathrm{~F}$-SMBT-1 radiotracer is a derivative of the ${ }^{18} \mathrm{~F}$-THK-5351 compound, and has shown high specificity to astrogliosis in an animal study recently [57]. The application of ${ }^{18} \mathrm{~F}-\mathrm{SMBT}-1$ PET in stroke patients may further substantiate the role of RA in PSCI occurrence.

This study had several limitations. First, although ${ }^{18} \mathrm{~F}$ THK-5351 can be used to quantify the RA extent in patients with stroke through the Z-map transformation method, there is still a need to validate the optimal Zscore threshold for demonstrating the RA extent. Our preliminary findings indicated that the $\mathrm{Z}$-score $>4$ threshold was most sensitive to PSCI manifestations. Second, ${ }^{18} \mathrm{~F}$-THK-5351 has a dual binding affinity to both tau protein and MAO-B, and such characteristic could limit the general applicability of ${ }^{18} \mathrm{~F}-\mathrm{THK}-5351$ in patients with stroke for neuroinflammation evaluation. Since our main interest was to explore the relationships between PSCI and neuroinflammation on ${ }^{18} \mathrm{~F}$-THK-5351 PET imaging, we only enrolled amyloid-negative stroke patients without tauopathy presentations to reduce the possibility of ${ }^{18} \mathrm{~F}$-THK-5351 binding to tau protein. The development of novel radiotracers specific to RA would be helpful to determine the cognitive impacts of strokerelated RA. Third, ${ }^{18} \mathrm{~F}$-THK-5351 PET was performed about 3 months after stroke in our study. As neuroinflammation effects on tissue remodeling are dynamic after neuronal injury, the RA presentations on ${ }^{18} \mathrm{~F}$-THK5351 PET imaging in acute stroke stage are yet to be determined [13]. Finally, this was a cross-sectional study with relatively small sample size. Future long-term follow-up studies should further investigate the dynamic relationships between RA and PSCI manifestations.

\section{Conclusion}

Stroke-induced ${ }^{18} \mathrm{~F}$-THK-5351 uptake signals could reflect the RA extent in amyloid-negative patients with first-ever stroke. Besides the cognitive effect from stroke lesion itself, stroke-induced neuroinflammation as measured by the total and ipsilateral Z-SUM scores of ${ }^{18} \mathrm{~F}$ THK-5351 PET imaging could further contribute to PSCI presentations.

\section{Supplementary information}

Supplementary information accompanies this paper at https://doi.org/10. 1186/s12974-020-01985-0.

\section{Additional file 1: Supplementary Table 1. Tests for}

neuropsychological function and stroke severity evaluation

Additional file 2: Supplementary Figure 1. The illustration of path analyses models. We used path analyses to evaluate whether Z-SUM scores of ${ }^{18} \mathrm{~F}-\mathrm{THK}-5351$ uptake intensity mediated the associations between stroke volume and cognitive performance. We selected cognitive functions showing significant correlations with both stroke volume and
Z-sum scores as the endogenous variables. Further, we adjusted for age, education, and depressive symptoms (a) and anxiety (b) co-variables.

Additional file 3: Supplementary Table 2. The comparisons of total Z-SUM scores between the presence and absence of vascular risk factors in stroke patients and healthy subjects

Additional file 4: Supplementary Table 3. Correlations of total Z-SUM scores with demographic, vascular and neurodegenerative features

Additional file 5: Supplementary Table 4. Associations of total ZSUM scores of ${ }^{18} \mathrm{~F}$-THK-5351 uptake intensity with cognitive function in model I

Additional file 6: Supplementary Table 5. Associations of total ZSUM scores of ${ }^{18} \mathrm{~F}-\mathrm{THK}-5351$ uptake intensity with cognitive function in model II

Additional file 7: Supplementary Table 6. Associations of total ZSUM scores of ${ }^{18} \mathrm{~F}-\mathrm{THK}$-5351 uptake intensity with cognitive function in model III

Additional file 8: Supplementary Table 7. Demographic data imaging findings and cognitive results between patients with left and right hemisphere stroke

Additional file 9: Supplementary Table 8. Correlations of contralateral Z-SUM scores with cognitive performance in patients with left and right hemisphere stroke

Additional file 10: Supplementary Figure 2. Path analyses of stroke effects on cognition via total or ipsilateral Z-SUM-4 scores after age, education and anxiety adjustment. Stroke volume contributes significantly to cognitive function as well as total and ipsilateral Z-SUM-4 scores in patients with either left-hemispheric $(a, b)$ or right-hemispheric $(c, d)$ stroke. Further, ipsilateral Z-SUM-4 scores can partly mediate language influence from stroke volume in patients with left-hemispheric stroke (b). Likewise, total and ipsilateral Z-SUM-4 scores can partly mediate executive influence from stroke volume in patients with right-hemispheric stroke (c, d)

Additional file 11: Supplementary Table 9. Effects of stroke volume and Z-SUM-4 score on cognitive function after adjustment for age, education and depressive symptoms (A D) and anxiety $(E \sim H)$

Additional file 12: Supplementary Table 10. Comparisons of the contralateral Z-SUM scores of stroke patients with the average Z-SUM scores of bilateral hemispheres of healthy subjects

Additional file 13: Supplementary Table 11. The correlations between total Z-SUM scores and cognitive function in ischemic stroke patients

Additional file 14: Supplementary Table 12. Clinical characteristics of patients with hemorrhagic and ischemic stroke

\section{Abbreviations}

AD: Alzheimer's disease; APOE $\varepsilon 4$ : Apolipoprotein E $\varepsilon 4 ;{ }^{11} \mathrm{C}-\mathrm{DED}:{ }^{11} \mathrm{C}$ deuterium-L-deprenyl; CDR-SOB: Sum of boxes of Clinical Dementia Rating; CT: Computed tomography; DWI: Diffusion-weighted imaging; DWML: Deep white matter leukoaraiosis; FLAIR: Fluid-attenuated inversion recovery; FWHM: Full width at half maximum; IADL: Instrumental Activities of Daily Living; IQCODE: Informant Questionnaire on Cognitive Decline in the Elderly; MAO-B: Monoamine oxidase-B; MMSE: Mini-Mental State Examination; MoCA: Montreal Cognitive Assessment; MPRAGE: Magnetization Prepared RApid Gradient Echo; MRI: Magnetic resonance imaging; MTA: Medial temporal atrophy; NIHSS: National Institutes of Health Stroke Scale; NPI: Neuropsychiatric Inventory; PET: Positron-emission tomography; PSCI: Post-stroke cognitive impairment; PSD: Post-stroke dementia; PVL: Periventricular leukoaraiosis; RA: Reactive astrogliosis; SUVR: Standardized uptake value ratio; T1W: T1-weighted; Z-SUM scores: Sum of ${ }^{18} \mathrm{~F}-\mathrm{THK}$-5351 uptake intensity $Z$ scores; $\mu^{\text {TP }}$ : Mean; $\sigma^{\text {TP. }}$ : Standard deviation

\section{Acknowledgments}

We thank Clinical Trial Center of Chang Gung Memorial Hospital for administrative assistance under support from the Ministry of Health and Welfare, Taiwan (MOHW104-TDU-B-212-113003; MOHW105-TDU-B-212133020; MOHW106-TDU-B-212-113005; MOHW107-TDU-B-212-123005). 


\section{Authors' contributions}

$\mathrm{KLH}$ wrote the initial draft, performed neurologic examinations, and took part in the data collection and analysis and scientific interpretation of data. ITH wrote a portion of the draft and carried out PET imaging analysis. MYH wrote a portion of the draft, conducted the cognitive evaluation, and took part in data analysis and critical review of the manuscript. JLH and YMW took part in MRI imaging analysis and scientific interpretation of data. $\mathrm{CCH}$, YJC, TYC, CHL, CHC, and KYW performed the data collection, neurologic examination and scientific interpretation of data. SPW, TCY, and NO conceptualized the study design and took part in the scientific interpretation of data. THL and KJL conceptualized the study design, took part in critical review of the manuscript, and edited the manuscript for content. All authors critically reviewed the manuscript and approved the final version for publication.

\section{Funding}

This study was supported from Ministry of Science and Technology, Taiwan (MOST 107-2314-B-182A-051, 106-2314-B-182A-026-MY3, 106-2314-B-182-017MY3), and Research Fund of Chang Gung Memorial Hospital (CPRPG3H0011, CMRPD1H0392, CORPG3J0342, BMRPD69, BMRP611).

\section{Availability of data and materials}

The datasets used and/or analyzed during the current study are available from the corresponding author on reasonable request.

\section{Ethics approval and consent to participate}

Ethical approval was obtained from the institutional review board at Linkou Chang Gung Memorial Hospital, Taiwan (IRB No. 103-7584A and 201601675A0) with the clinical trials registered to Taiwan Food and Drug Administration (1040025953 and 1066015148) and Center of Drug Evaluation (104IND06124 and 106IND03071). The study protocol and procedure were complied with the 1964 Helsinki Declaration and its later amendments or comparable ethical standards. Informed consent was obtained from all individual participants included in the study.

\section{Consent for publication}

Not applicable.

\section{Competing interests}

The authors declare that they have no competing interests.

\section{Author details}

'Department of Neurology, Linkou Chang Gung Memorial Hospital, and College of Medicine, Chang Gung University, No. 5, Fuxing St., Guishan Taoyuan, Taiwan. ${ }^{2}$ Department of Nuclear Medicine and Molecular Imaging Center, Linkou Chang Gung Memorial Hospital, No. 5, Fuxing St., Guishan, Taoyuan, Taiwan. ${ }^{3}$ Healthy Aging Research Center and Department of Medical Imaging and Radiological Sciences, College of Medicine, Chang Gung University, Taoyuan, Taiwan. ${ }^{4}$ Graduate Institute of Behavioral Sciences, Chang Gung University, Taoyuan, Taiwan. ${ }^{5}$ Department of Neurology, New Taipei Municipal TuCheng Hospital, Chang Gung Memorial Hospital, Chang Gung University, New Taipei City, Taiwan. ${ }^{6}$ Taipei Medical University, College of Humanities and Social Sciences, Graduate Institute of Humanities in Medicine and Research Center for Brain and Consciousness, Shuang Ho Hospital, Taipei, Taiwan. 'Department of Radiology, Chang Gung Memorial Hospital, Taoyuan, Taiwan. ${ }^{8}$ Department of Psychiatry, Chang Gung Memorial Hospital, Taoyuan, Taiwan. ${ }^{9}$ Division of Neuro-imaging, Institute of Development, Aging and Cancer, Tohoku University, Sendai, Japan. ${ }^{10}$ Division of Pharmacology, Faculty of Medicine, Tohoku Medical and Pharmaceutical University, Sendai, Japan.

\section{Received: 3 August 2020 Accepted: 5 October 2020}

\section{Published online: 17 October 2020}

\section{References}

1. Mijajlović MD, et al. Post-stroke dementia - a comprehensive review. BMC Med. 2017;15:11

2. Henon H, Pasquier F, Leys D. Poststroke dementia. Cerebrovasc Dis. 2006;22: 61-70.

3. Tuladhar AM, de Leeuw FE. Poststroke dementia--what's in a name? Nat Rev Neurol. 2010;6:63-4.
4. Leys D, et al. Poststroke dementia. Lancet Neurol. 2005;4:752-9.

5. Thiel A, et al. Amyloid burden, neuroinflammation, and links to cognitive decline after ischemic stroke. Stroke. 2014;45:2825-9.

6. Kim H, et al. Assessment of extent and role of tau in subcortical vascular cognitive impairment using 18F-AV1451 positron emission tomography imaging. JAMA Neurol. 2018:999-1007.

7. Rothenburg LS, et al. The relationship between inflammatory markers and post stroke cognitive impairment. J Geriatr Psychiatry Neurol. 2010;23:199_ 205.

8. Narasimhalu K, et al. Inflammatory markers and their association with post stroke cognitive decline. Int J Stroke. 2013;10:513-8.

9. Thiel A, et al. The temporal dynamics of poststroke neuroinflammation: a longitudinal diffusion tensor imaging-guided PET study with 11C-PK11195 in acute subcortical stroke. J Nucl Med. 2010;51:1404-12.

10. Okamura $\mathrm{N}$, et al. PET imaging of tau pathology in mild cognitive impairment and Alzheimer's disease with [18F]THK-5351. J Nucl Med. 2015; 56:138.

11. Ng KP, et al. Monoamine oxidase B inhibitor, selegiline, reduces $18 \mathrm{~F}-$ THK5351 uptake in the human brain. Alzheimers Res Ther. 2017:9:25.

12. Harada R, Ishiki A. Correlations of 18F-THK5351 PET with post-mortem burden of tau and astrogliosis in Alzheimer's disease. J Nucl Med. 2018;59: 671-4.

13. Burda JE, Sofroniew MV. Reactive gliosis and the multicellular response to CNS damage and disease. Neuron. 2014;81:229-48.

14. Ishibashi $\mathrm{K}$, et al. Potential use of 18F-THK5351 PET to identify wallerian degeneration of the pyramidal tract caused by cerebral infarction. Clin Nucl Med. 2017;42:e523-e4.

15. Huang $\mathrm{K}-\mathrm{L}$, et al. Visualization of ischemic stroke-related changes on 18FTHK-5351 positron emission tomography. EJNMMI Res. 2018:8:62.

16. Fuh $J-L$, et al. The Informant Questionnaire on Cognitive Decline in the Elderly (IQCODE) as a screening tool for dementia for a predominantly illiterate Chinese population. Neurology. 1995:45:92-6.

17. Srikanth VK, et al. Long-term cognitive transitions, rates of cognitive change, and predictors of incident dementia in a population-based first-ever stroke cohort. Stroke. 2006;37:2479-83.

18. Huang K-L, et al. Asymmetric cerebrovascular collateral supply affects cognition in patients with unilateral carotid artery stenosis. Curr Neurovasc Res. 2017:14:347-58.

19. Huang $K-L$, et al. The correlation of asymmetrical functional connectivity with cognition and reperfusion in carotid stenosis patients. Neuroimage Clin. 2018:20:476-84

20. Tang WK, et al. Can IQCODE detect poststroke dementia? Int J Geriatr Psychiatry. 2003;18:706-10.

21. Srikanth VK, et al. Increased risk of cognitive impairment 3 months after mild to moderate first-ever stroke: a community-based prospective study of nonaphasic English-speaking survivors. Stroke. 2003;34:1136-43.

22. Marshall RS, et al. Cerebral hemodynamics and cognitive impairment: baseline data from the RECON trial. Neurology. 2012;78:250-5.

23. Becker JA, et al. Amyloid- $\beta$ associated cortical thinning in clinically normal elderly. Ann Neurol. 2011;69:1032-42

24. Scheltens $P$, van de Pol L. Atrophy of medial temporal lobes on MRI in "probable" Alzheimer's disease and normal ageing: diagnostic value and neuropsychological correlates. J Neurol Neurosurg Psychiatry. 2012:83:1038-40.

25. Huang $\mathrm{K}-\mathrm{L}$, et al. Correlation between visual association memory test and structural changes in patients with Alzheimer's disease and amnestic mild cognitive impairment. J Formos Med Assoc. 2019;118:1325-32.

26. Fazekas F, et al. MR signal abnormalities at 1.5 T in Alzheimer's dementia and normal aging. Am J Roentgenol. 1987;149:351-6.

27. Cordonnier $C_{\text {, et }}$ al. Improving interrater agreement about brain microbleeds: development of the Brain Observer MicroBleed Scale (BOMBS). Stroke. 2009;40:94-9.

28. Staals J, et al. Stroke subtype, vascular risk factors, and total MRI brain smallvessel disease burden. Neurology. 2014;83:1228-34.

29. Doubal Fergus N, et al. Enlarged perivascular spaces on MRI are a feature of cerebral small vessel disease. Stroke. 2010;41:450-4.

30. Huang C-C, et al. Tau PET with 18F-THK-5351 Taiwan patients with familial Alzheimer's disease with the APP p.D678H mutation. Front Neurol. 2019;10: 503. https://doi.org/10.3389/fneur.2019.00503.

31. Johnson KA, et al. Florbetapir (F18-AV-45) PET to assess amyloid burden in Alzheimer's disease dementia, mild cognitive impairment, and normal aging. Alzheimers Dement. 2013;9:572-83. 
32. Minoshima S, et al. A diagnostic approach in Alzheimer's disease using three-dimensional stereotactic surface projections of fluorine-18-FDG PET. J Nucl Med. 1995;36:1238-48.

33. Fällmar $D$, et al. Arterial spin labeling-based Z-maps have high specificity and positive predictive value for neurodegenerative dementia compared to FDG-PET. Eur Radiol. 2017;27:4237-46.

34. Maillard $P$, et al. White matter hyperintensities and their penumbra lie along a continuum of injury in the aging brain. Stroke. 2014;45:1721-6.

35. Simats A, García-Berrocoso T, Montaner J. Neuroinflammatory biomarkers: from stroke diagnosis and prognosis to therapy. Biochim Biophys Acta. 1862;2016:411-24.

36. Barreto $G E$, et al. Astrocyte proliferation following stroke in the mouse depends on distance from the infarct. PLoS One. 2011;6:e27881.

37. Anrather J, ladecola C. Inflammation and stroke: an overview. Neurotherapeutics. 2016:13:661-70

38. Carter SF, et al. Astrocyte biomarkers in Alzheimer's disease. Trends Mol Med. 2019;25:77-95.

39. Silver J, Edwards MA, Levitt P. Immunocytochemical demonstration of early appearing astroglial structures that form boundaries and pathways along axon tracts in the fetal brain. J Comp Neurol. 1993;328:415-36.

40. Edison P, Brooks DJ. Role of neuroinflammation in the trajectory of Alzheimer's disease and in vivo quantification using PET. J Alzheimers Dis. 2018;64:S339-S51.

41. ter Telgte $\mathrm{A}$, et al. Cerebral small vessel disease: from a focal to a global perspective. Nat Rev Neurol. 2018;14:387-98.

42. Reijmer YD, et al. The effect of lacunar infarcts on white matter tract integrity. Stroke. 2013;44:2019-21.

43. Duering $M$, et al. Acute infarcts cause focal thinning in remote cortex via degeneration of connecting fiber tracts. Neurology. 2015;84:1685-92.

44. Gauberti M, De Lizarrondo SM, Vivien D. The "inflammatory penumbra" in ischemic stroke: from clinical data to experimental evidence. Eur Stroke J. 2016;1:20-7.

45. Horváth $\mathrm{E}$, et al. Ischemic damage and early inflammatory infiltration are different in the core and penumbra lesions of rat brain after transient focal cerebral ischemia. J Neuroimmunol. 2018;324:35-42.

46. Hesse $C$, et al. Transient increase in total tau but not phospho-tau in human cerebrospinal fluid after acute stroke. Neurosci Lett. 2001;297:187-90.

47. Hesse C, et al. Cerebrospinal fluid markers for Alzheimer's disease evaluated after acute ischemic stroke. J Alzheimers Dis. 2000;2:199-206.

48. Jayaraj RL, et al. Neuroinflammation: friend and foe for ischemic stroke. J Neuroinflammation. 2019;16:142.

49. Tschoe C, et al. Neuroinflammation after intracerebral hemorrhage and potential therapeutic targets. J Stroke. 2020;22:29-46.

50. Mestriner RG, et al. Astrocyte morphology after ischemic and hemorrhagic experimental stroke has no influence on the different recovery patterns. Behav Brain Res. 2015;278:257-61.

51. Fang $M$, et al. Effect of inflammation on the process of stroke rehabilitation and poststroke depression. Front Psychiatry. 2019;10.

52. Pendlebury ST, Rothwell PM. Prevalence, incidence, and factors associated with pre-stroke and post-stroke dementia: a systematic review and metaanalysis. Lancet Neurol. 2009;8:1006-18.

53. Wen $\mathrm{H}$, et al. Inflammatory signaling in post-stroke fatigue and depression Eur Neurol. 2018;80:138-48.

54. Lin JH, et al. Prediction of poststroke dementia. Neurology. 2003;61:343-8.

55. Gerhard A, et al. Evolution of microglial activation in patients after ischemic stroke: a [11C](R)-PK11195 PET study. Neurolmage. 2005;24:591-5.

56. Tronel $\mathrm{C}$, et al. Molecular targets for PET imaging of activated microglia: the current situation and future expectations. Int J Mol Sci. 2017;18:802.

57. Harada $\mathrm{R}$, et al. 18F-SMBT-1: a selective and reversible positron-emission tomography tracer for monoamine oxidase-B imaging. J Nucl Med. 2020: jnumed.120.244400. https://doi.org/10.2967/jnumed.120.244400. Epub ahead of print.

\section{Publisher's Note}

Springer Nature remains neutral with regard to jurisdictional claims in published maps and institutional affiliations.

Ready to submit your research? Choose BMC and benefit from:

- fast, convenient online submission

- thorough peer review by experienced researchers in your field

- rapid publication on acceptance

- support for research data, including large and complex data types

- gold Open Access which fosters wider collaboration and increased citations

- maximum visibility for your research: over $100 \mathrm{M}$ website views per year

At BMC, research is always in progress.

Learn more biomedcentral.com/submissions 